\title{
Vinculando educación matemática y sostenibilidad: implicaciones para la formación inicial de maestros como herramienta de transformación social
}

\author{
Ángel Alsina \\ Departamento de Didácticas Específicas, Facultad de Educación y Psicología. \\ Universidad de Girona. España. angel.alsina@udg.edu \\ ORCID: https://orcid.org/0000-0001-8506-1838 \\ M. Teresa Calabuig \\ Departamento de Didácticas Específicas, Facultad de Educación y Psicología. \\ Universidad de Girona. España. teresa.calabuig@udg.edu \\ ORCID: https://orcid.org/0000-0003-2026-3732
}

[Recibido: 13 Noviembre 2018. Revisado: 11 Enero 2019. Aceptado: 5 Febrero 2019]

Resumen: La finalidad de este estudio es avanzar hacia la definición de un modelo que permita caracterizar la formación inicial del profesorado de matemáticas en sintonía con la sostenibilidad, para que los futuros maestros puedan proporcionar una educación matemática a sus alumnos que permita afrontar con mayor éxito las demandas sociales contemporáneas. Con este propósito, se ha realizado un estudio exploratorio con 30 informantes (10 formadores de maestros, 10 maestros en activo y 10 futuros maestros de Educación Primaria) a los que se les ha administrado el Cuestionario EMS "Educación Matemática y Sostenibilidad", previamente validado. Los datos obtenidos han permitido definir un modelo formado por cinco dimensiones: 1) vínculos con el entorno; 2) conocimientos didáctico-disciplinares; 3) pensamiento reflexivo y crítico; 4) necesidades de los niños para aprender; 5) ambientalización curricular. La incorporación de estas cinco dimensiones puede empoderar el desarrollo profesional de los futuros maestros de matemáticas de Educación Primaria para que contribuyan a dar una respuesta eficaz a los retos sociales propios de un mundo complejo.

Palabras clave: Educación matemática; Educación para la Sostenibilidad; Formación inicial de maestros; Desarrollo Profesional; Educación Primaria.

Linking mathematics education and sustainability: implications for teachers' initial training as a tool for social transformation.

Abstract: The aim of this study is to advance towards the definition of a model that allows to characterize teachers' initial training of mathematics according to sustainability, so that future teachers can provide a mathematical education to their students that allows them to face with greater success contemporary social demands. For this purpose, an exploratory study has been carried out with 30 informants (10 teacher trainers, 10 active teachers and 10 future teachers of Primary Education) who have been administered the previously validated EMS "Mathematics and Sustainability Education" Questionnaire. The data obtained have allowed to define a model composed by five dimensions: 1) links with the environment; 2) didactic-disciplinary knowledge; 3) reflective and critical thinking; 4) Children's needs to learn; 5) curricular environmentalization. The incorporation of these five dimensions can empower the professional development of pre-service teachers so that they contribute to give an effective answer to the social challenges of a complex world.

Keywords: Mathematics education; Education for Sustainability; Initial teacher training; Professional development; Primary education. 
Para citar este artículo: Alsina, A. y Calabuig, M. T. (2019) Vinculando educación matemática y sostenibilidad: implicaciones para la formación inicial de maestros como herramienta de transformación social. Revista de Educación Ambiental y Sostenibilidad 1(1), $1203 . \quad$ doi: 10.25267/Rev_educ_ambient_sostenibilidad.2019.v1.i1.1203

\section{Introducción}

Uno de los ámbitos temáticos más prolíficos de la investigación en educación matemática durante los últimos años se centra en el desarrollo profesional del profesor (Llinares, 2008). El interés por cuestiones relativas a las creencias de los profesores de matemáticas, en los intentos de comprender mejor los procesos de aprender y enseñar y de desarrollo profesional se ha puesto de manifiesto a través de grupos de investigadores que intentan compartir sus estudios, tanto a nivel internacional como nacional, como el WG Research on the Psychology of Mathematics Teacher Development en el International Group for the Psychological Mathematics Education o bien el Grupo de Investigación Conocimiento y Desarrollo Profesional del Profesor dentro de la Sociedad Española de Investigación en Educación Matemática. Los temas de estudio de estos grupos se centran, principalmente, en el desarrollo, comunicación y estudio de cuestiones y esquemas de índole conceptual en varias agendas de investigación sobre el profesor de matemáticas; explorar metodologías de investigación innovadoras en los estudios sobre el profesor de matemáticas y clarificar las bases teóricas de estas metodologías o bien desarrollar estándares de calidad de la investigación.

En este marco, nuestro interés se focaliza en indagar acerca de qué elementos puede aportar la sostenibilidad para mejorar la formación inicial del profesorado de matemáticas. Este interés se fundamenta en el hecho de que el momento histórico actual está caracterizado por unas circunstancias socioculturales que determinan la manera más adecuada de encarar la educación: la globalización del mundo, las crisis ambiental y económica y por lo tanto también social, han hecho que grupos de trabajo de muchas universidades del mundo y la misma Organización de las Naciones Unidas, a través de la UNESCO, haya tomado la sostenibilidad como referente y guía para la educación del siglo XXI (Robinson, Astorga, Oksanen y Tardo, 1993). Desde este prisma, tener presente criterios de sostenibilidad en el diseño de los curricula académicos de todos los campos del saber y de todos los niveles educativos se convierte en una necesidad de compromiso social que contribuye a hacer crecer la sintonía entre qué y cómo se trabaja en los centros educativos y qué demanda la sociedad.

En este contexto nos planteamos distintos interrogantes, como por ejemplo ¿cómo puede contribuir la formación de maestros de matemáticas a las exigencias sociales del S. XXI?, ¿la educación matemática puede, por ella misma, dar una respuesta global a todas estas exigencias o, por el contrario, es necesario considerar los avances de investigación de otros campos de conocimiento? y, más en concreto, y en sintonía con los planteamientos de la UNESCO, ¿qué puede aportar la sostenibilidad a la educación matemática para poder planificar y gestionar una formación inicial de maestros que contribuya a dar una respuesta eficaz a los retos sociales contemporáneos? Desde este prisma, se presenta un estudio cuya finalidad consiste en avanzar hacia la definición de un modelo que permita caracterizar la formación inicial del profesorado de matemáticas en sintonía con la sostenibilidad, a partir de los últimos avances tanto en 
cuestiones sobre el desarrollo profesional del profesorado de matemáticas como de la educación para la sostenibilidad, y considerando también los datos de investigaciones previas en las que ya se establecieron criterios para definir un perfil de maestro de matemáticas sensible con este reto social (Calabuig, 2017; Calabuig, Alsina y Geli, 2017).

\section{La formación de maestros de matemáticas como herramienta de transformación social}

En este artículo se asume que lo que hará de la formación de maestros una herramienta de cambio, de regulación y de renovación social será que ella misma sea capaz de cambiar, regularse y renovarse. Tal como señalan Gutiérrez-Pérez y PeralesPalacios (2012, p. 7):
A las novedades y sorpresas que cada época aporta, se añaden los cambios disciplinares, las tensiones conceptuales, los adelantos de la investigación sistemática. La educación contemporánea tendría que tener recursos capaces de atender estas nuevas demandas sociales y los educadores estar dotados de capacidad de respuesta suficiente para afrontar los retos, estar a la altura de las circunstancias en la recomposición crítica de las realidades cambiantes y favorecer el desarrollo de habilidades en las nuevas generaciones para el ejercicio de la reconstrucción continuada de su realidad.

Calabuig (2017) señala que para avanzar en esta dirección, esta formación tiene que encarar determinados retos que la acerquen más a la formación integral de la persona. De este modo, los maestros dispondrán de más herramientas para ser ellos mismos un agente de cambio. En este sentido, una vía para definir estos retos en la formación inicial del profesorado es enfocando su formación desde el aprendizaje reflexivo (Korthagen, 2001; Esteve, Melief y Alsina, 2010) y desde la ambientalización curricular (Junyent, Geli y Arbat, 2003; CRUE_CADEP, 2016); el primero aporta herramientas para fomentar procesos de autorregulación que favorecen el desarrollo profesional del profesorado a partir de la relación entre la práctica y la teoría; y el segundo permite adaptar el plan de estudios en base a criterios de desarrollo sostenible (Figura 1).

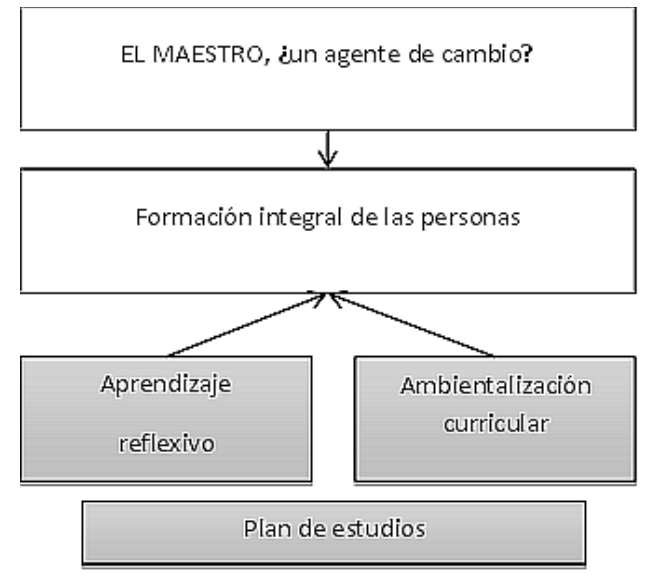

Figura 1. El maestro como agente de cambio 


\section{El aprendizaje reflexivo en la formación inicial de maestros de matemáticas}

Hace ya varias décadas, Kilpatrick (1988, p. 204) señaló que parece que alguna cosa no funciona teniendo a un grupo diciendo qué hacer y otro haciéndolo, aludiendo de forma explícita a los formadores de maestros por un lado y a los futuros maestros por otro, argumento que ha sido apoyado por otros reconocidos investigadores del ámbito de la educación matemática, como por ejemplo Freudenthal (1991). En este sentido, Melief, Tigchelaar y Korthagen (2010) indican que uno de los mayores problemas de esta perspectiva es que se parte del supuesto que el aprendizaje se desarrolla de manera más o menos lógico-racional, es decir, los formadores de maestros intentan precisar el camino del aprendizaje previamente, pero a menudo los aprendices de maestro se encuentran con problemas y preguntas que los formadores no pueden prever con detalle. Considerando estas limitaciones, defienden un modelo de formación que integre a los futuros maestros con sus experiencias personales, los conocimientos teóricos y la práctica del aula, y ello a través de la reflexión. De acuerdo con Korthagen (2001), el proceso ideal del aprendizaje reflexivo consiste en una alternancia entre acción y reflexión a través de cinco fases, que determinan el modelo ALACT: acción o experiencia; mirar hacia atrás (hacia la acción); tomar conciencia y determinar los aspectos importantes de la propia actuación; buscar y preparar comportamientos alternativos para actuar; y comprobar su eficacia en una nueva situación, lo que ofrece, otra vez, una nueva experiencia y, por lo tanto, es el punto de partida de un nuevo ciclo de reflexión (véase Figura 2).

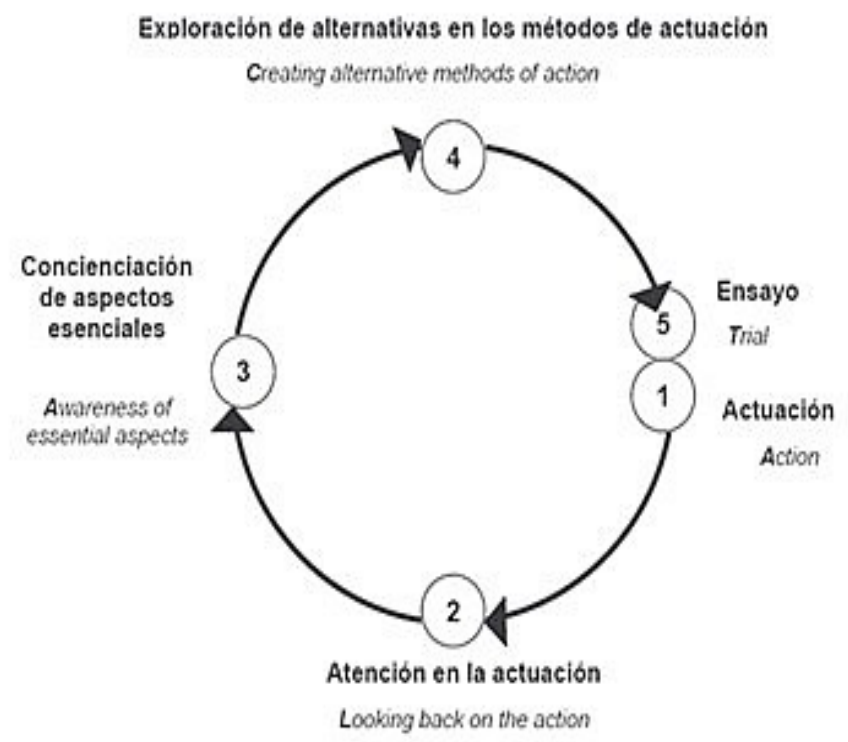

Figura 2. Modelo ALACT (Korthagen, 2010:91).

Alsina $(2007 ; 2010)$, considerando estos planteamientos, adapta el modelo ALACT a la formación inicial del profesorado de matemáticas. En dicha adaptación, se consideran cuatro fases: 1) toma de consciencia de las creencias, experiencias y conocimientos previos; 2) interacción con los demás, con uno mismo y con la teoría para coconstruir nuevos conocimientos; 3) contraste entre los nuevos conocimientos y las creencias, experiencias y conocimientos previos; 4) reconstrucción. En síntesis, se trata de un proceso de deconstrucción de conocimientos implícitos previos que son un obstáculo para el desarrollo profesional y de coconstrucción y reconstrucción de los nuevos conocimientos profesionales que empoderan un perfil docente propio. 
Para Llinares (2012), una de las características que define el proceso de llegar a tener un conocimiento experto es la manera en la que un maestro identifica lo que es relevante en una situación de enseñanza y lo que conocen para interpretarlo. Se trata, en definitiva, de dilucidar cómo los estudiantes para maestro aprenden a usar sus conocimientos de matemáticas y de didáctica de las matemáticas para dotar de sentido a las situaciones de enseñanza que les permita actuar de manera pertinente. Para dar cuenta de esta habilidad, este autor y su equipo usan la expresión mirar profesionalmente. Llinares (2018, p. 40) indica que con este término, en sintonía con el modelo del aprendizaje reflexivo,

Se intenta recoger la manera en la que los profesores piensan sobre su práctica (con un énfasis en la práctica reflexiva) para comprenderla y mejorarla. Pero, además permite centrar la atención sobre la manera en la que los estudiantes para maestro aprenden a usar el conocimiento de matemáticas y de didáctica de las matemáticas para comprender las situaciones de enseñanza y actuar como maestros. De esta manera mirar profesionalmente consiste en usar el conocimiento para determinar lo que es relevante en una situación de enseñanza y establecer relaciones con ideas teóricas (conocimiento teórico) para apoyar las decisiones en relación a qué hacer a continuación (Sherin, 2007). El adjetivo profesional intenta subrayar el hecho del uso de manera consciente de un conocimiento teórico que permite a los profesores comprender las situaciones de enseñanzaaprendizaje.

Los planteamientos tanto del aprendizaje reflexivo como de la perspectiva mirar profesionalmente, por su naturaleza, pueden aportar herramientas eficaces a la formación de maestros de matemáticas que contribuyan a la transformación social.

\section{La ambientalización de los currículos de matemáticas.}

Con el propósito de incorporar la sostenibilidad en los procesos de formación universitaria, la red Copernicus-Alliance de universidades europeas para la sostenibilidad trabaja desde 1993 con este objetivo, con especial énfasis en la formación del profesorado de Educación Primaria y Secundaria (Copernicus-Alliance, 2016). A nivel español, la red de Ambientalización Curricular de los Estudios Superiores (ACES) ha realizado múltiples aportaciones desde su creación en el año 2002 para la incorporación de la sostenibilidad como una competencia transversal (Junyent, Geli y Arbat, 2003). Aquel mismo año, dentro de la Conferencia de Rectores de Universidades Españolas (CRUE) se formó el Grupo de Sostenibilidad Curricular de la Comisión Sectorial de Calidad Ambiental, Desarrollo Sostenible y Prevención de Riesgos (CADEP), que vela por la calidad ambiental, el desarrollo sostenible y la prevención de riesgos. Desde el inicio aprobó una serie de directrices para la introducción de la sostenibilidad en los curricula universitarios, que posteriormente fueron revisados (CRUE-CADEP, 2016). También el Departamento de Medio Ambiente de la Generalitat de Cataluña puso en marcha un proyecto para adaptar la ambientalización curricular a los centros de educación primaria y secundaria adheridos a la red de escuelas verdes (Geli, Junyent, Medir y Padilla, 2006) puesto que, de acuerdo con Calabuig (2017), en el proceso de ambientalización de los curricula los maestros tienen un papel clave, ya que son ellos los últimos responsables de vehicular la incorporación de criterios de sostenibilidad en el curriculum. 
En este sentido, a continuación se enumeran diversos retos que debería asumir la educación superior, incluida la formación inicial de maestros de matemáticas, para llevar a cabo una ambientalización curricular adecuada (Barañano y Comisión RSU, 2011; Copernicus-Alliance, 2016; CRUE_CADEP, 2016; Geli et al., 2006; Goñi, 2010; Junyent, Geli y Arbat, 2003; Schmittau, 2003; UNESCO, 2004).

- Introducir de manera efectiva las ideas de la educación para la sostenibilidad en el curriculum: se debería impregnar el curriculum de criterios de sostenibilidad para poder llevar a cabo una formación capaz de afrontar con naturalidad y normalidad la complejidad del momento actual.

- Seleccionar el contenido del curriculum con criterios de sostenibilidad: si se diseña el curriculum a través de la tradición selectiva se conseguirá que esté diseñado de manera adecuada para un presente y un futuro marcados por un pasado histórico que define las necesidades sociales.

- Introducir cambios curriculares a largo plazo: una de las voluntades de la ambientalización curricular es que los cambios que se introduzcan en el curriculum puedan responder a necesidades educativas y profesionales no sólo del momento actual, sino en escenarios sociales y culturales posteriores.

- Introducir de manera efectiva las ideas de la educación para la sostenibilidad en la realidad del aula universitaria: se debería facilitar la práctica docente para que no encuentre obstáculos innecesarios para introducir de manera efectiva la educación para la sostenibilidad en las aulas.

- Ofrecer una formación general, diversa y holística: los futuros maestros deberían tener acceso a una formación rica y variada que los convierta en profesionales y ciudadanos más preparados para desarrollar su trabajo en conexión con el desarrollo sostenible.

- Vincular el uso del conocimiento a la responsabilidad social: el conocimiento y la educación superior deberían estar al servicio de la sociedad para innovar a favor de su desarrollo.

- Facilitar el acceso universal a la educación superior: para construir una sociedad sólida hacen falta ciudadanos que hayan tenido la oportunidad de formarse en el campo académico y profesional que tanto ellos como la sociedad necesiten.

- Velar por una educación superior que mire más allá de sus fronteras: complejidad, globalización, interacción, socialización, etc. son cuestiones imprescindibles en la formación del profesorado.

- Ambientalizar tanto la docencia como la investigación: si, como es deseable, los cambios en la formación inicial de maestros están influenciados por los resultados de la investigación, una ambientalización de la primera se conseguirá a través de la ambientalización de la segunda.

- Implicar a toda la comunidad universitaria: la calidad de la educación superior no es responsabilidad sólo del profesorado, sino de toda la comunidad educativa. Todos, desde su lugar y su visión profesional, deberían ayudar a diseñar una educación superior al servicio de la sociedad. 
- Abrir la universidad a la comunidad: el rechazo de la endogamia formativa permite que sus graduados se formen de acuerdo con su objetivo real, que es la sociedad.

- Formar ciudadanos críticos, preparados y dispuestos a actuar: si la universidad prepara a los estudiantes para que sean críticos, también tiene que asumir la responsabilidad y el trabajo de enseñarles a actuar de acuerdo con lo que ellos critiquen.

- Aprender a hacer un uso responsable de las TIC en las aulas: las nuevas tecnologías permiten acceder a información de una manera que años atrás hubiera sido impensable, pero no deberían sustituir la experimentación y el contacto directo con el mundo físico, social y cultural.

- Preparar a los futuros maestros para que sepan adaptarse a diferentes situaciones a medida que el aprendizaje progresa: en la formación inicial se distinguen dos tipos de discurso: el didáctico y el disciplinario. Los futuros maestros deben estar formados en los dos campos porque a medida que el aprendizaje vaya avanzando sepan ir adaptando el discurso didáctico a las nuevas necesidades educativas.

- Preparar a todos los graduados para el saber (conocimiento), el saber hacer (capacidades), el saber ser (habilidades), el saber estar (actitudes) y el saber actuar (movilizar recursos personales).

González (2012, p. 23) indica que asumir todos estos retos implica:

[...] dirigir los esfuerzos hacia el aprendizaje de principios, criterios, capacidades y valores. De otro modo, el currículum y la institución escolar en su conjunto se convertirán cada vez más en un cliente dócil de los intereses que circulan sin ninguna restricción por el espacio global.

Con base a estos antecedentes, se ha realizado un estudio con 30 informantes con el propósito de avanzar hacia la definición de un modelo que permita caracterizar la formación inicial del profesorado de matemáticas en sintonía con la sostenibilidad.

\section{Metodología}

Se ha llevado a estudio exploratorio en tres fases:

1. Diseño, construcción y validación del Cuestionario Educación Matemática y Sostenibilidad (Cuestionario EMS). Se trata de un cuestionario con 14 ítems sobre aspectos relacionados con lo que debe saber, saber hacer y saber valorar un maestro de matemáticas para que su acción guíe hacia el desarrollo sostenible (para una revisión, consultar Calabuig 2017, p. 115-120).

2. Aplicación del Cuestionario EMS a 30 informantes, a través del correo electrónico y de Google Drive. Los informantes pertenecen a tres colectivos diferentes (10 de cada colectivo): formadores de maestros, maestros en activo y futuros maestros de Educación Primaria. Los criterios de selección de los informantes han sido que fueran cercanos geográficamente y afines al contexto cultural en el que se realiza el estudio; y que tuvieran la formación y sensibilidad necesarias para poder opinar sobre educación matemática o educación para la sostenibilidad. 
3. Análisis de los datos obtenidos, a través de una categorización mixta: inductiva en las preguntas con respuestas abiertas; y deductiva en las preguntas en las que se ofrecían opciones de respuesta a los informantes.

Seguidamente se presenta, a modo de ejemplo, la categorización realizada en dos de las catorce preguntas del Cuestionario-EMS (extraído de Calabuig, Alsina y Geli, 2017):

Pregunta 1: Diga tres factores que hacen que una persona quiera aprender matemáticas.

Las categorías que se establecieron a partir de las repuestas recibidas fueron: 1 . Sociales y de relación con: a) uno mismo; b) el mundo y la sociedad; c) el maestro y/o sistema escolar; d) las matemáticas; 2. Profesionales y/o de estudio; 3. Procesos mentales y de comprensión: a) del mundo; b) de hechos matemáticos. Para cada categoría se ofrecía una breve aclaración sobre a qué hacían referencia y se mostraban respuestas concretas dadas por los informantes (Tabla 1).

Tabla 1. Ejemplo de categorización y de respuestas a la pregunta 1 referente al deseo de aprender matemáticas

\begin{tabular}{|c|c|c|}
\hline Categoría & Hace referencia a... & Ejemplos de respuestas \\
\hline \multicolumn{3}{|c|}{ Sociales y de relación con... } \\
\hline Uno mismo & $\begin{array}{l}\text { La autosatisfacción cuando se } \\
\text { resuelve o se entiende un reto } \\
\text { matemático }\end{array}$ & $\begin{array}{l}<<\text { La sorpresa y los sentimientos } \\
\text { positivos que experimentamos } \\
\text { cuando entendemos las } \\
\text { matemáticas }>> \\
<<\text { Nos hacen pensar y sentir, } \\
\text { hacen que crezcamos }>>\end{array}$ \\
\hline
\end{tabular}

Pregunta 6: Indique tres tipos de actividades no académicas que permitan al maestro mantener actualizada su capacidad para trabajar la EDM en conexión con la EDS.

1. Mantenerse informado.

2. Participar en tertulias sobre temas de actualidad.

3. Relacionarse con edades y/o grupos diferentes.

4. Viajar.

5. Leer novela histórica.

6. Leer artículos de opinión.

7. Aprender cosas nuevas.

8. Leer en general.

9. Estudiar el tema.

10. Comunicación por redes sociales.

En este caso, las categorías se establecieron de antemano con la formulación de la propia pregunta.

\section{Resultados}

En lo que sigue se presentan los resultados correspondientes a los 14 ítems del Cuestionario EMS. Cabe señalar que, aunque se dispone de los datos parciales de cada 
grupo de informantes, se presentan exclusivamente los resultados globales dado que lo que se pretende es caracterizar un modelo definido entre todos.

\section{Deseo de aprender matemáticas}

Con esta primera pregunta se pretende recoger el interés de los informantes acerca de los factores que intervienen en el gusto hacia las matemáticas (ver figura 3).

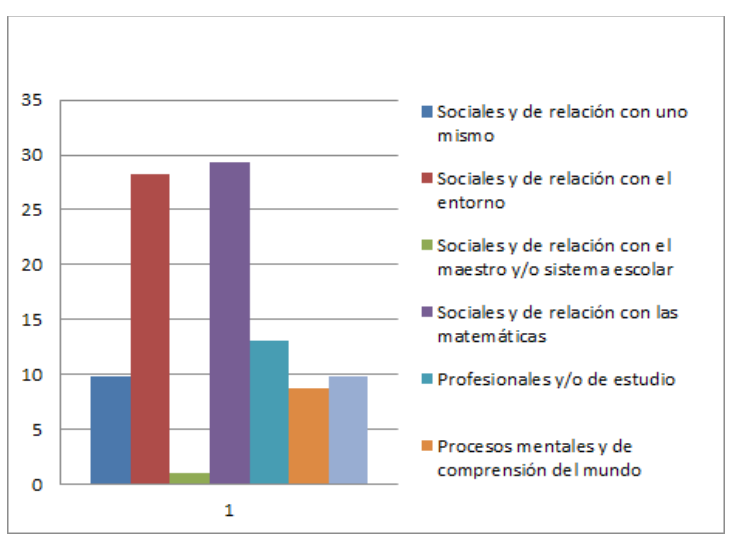

Figura 3. Deseo de aprender matemáticas

De acuerdo con las respuestas obtenidas, el profesorado debería estar capacitado para fomentar que los estudiantes establezcan una buena relación entre las matemáticas y el mundo que lo rodea y entre ellos mismos y la esencia de las matemáticas. Así mismo, debería ofrecer una buena base de educación matemática para etapas posteriores de estudios así como para facilitar el estudio de otras disciplinas en el momento presente.

\section{Diversidad sociocultural}

Los datos que se muestran en la figura 4 muestran cómo los informantes piensan que se puede aprovechar la diversidad sociocultural existente en las aulas a favor de la educación matemática.

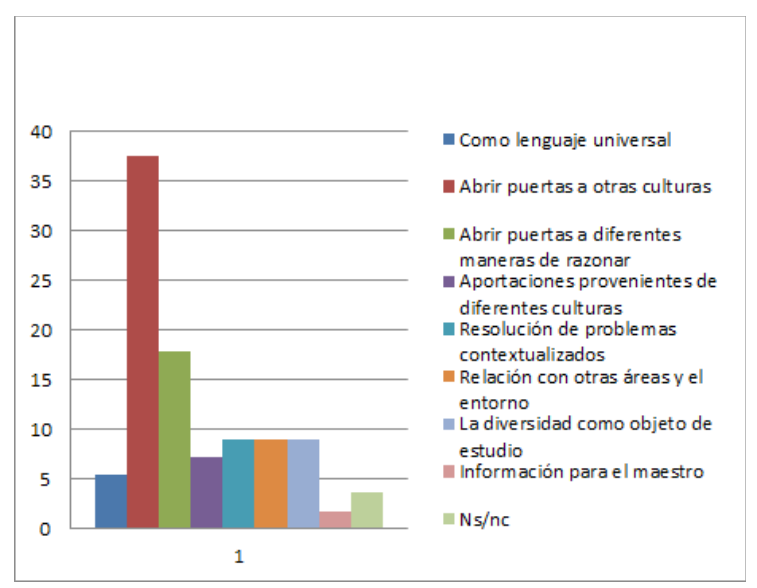

Figura 4. Diversidad sociocultural

Según las respuestas obtenidas, el profesorado debería estar capacitado principalmente para respetar, hacer respetar, valorar e impulsar personalmente y académicamente diferentes maneras de razonar un mismo hecho matemático, junto 
con impulsar la colaboración en el aula de estas diferentes maneras de razonar para conseguir un objetivo común.

\section{Responsabilidad y compromiso social}

Con este ítem se pretende poner de manifiesto que la educación matemática puede ayudar a promover la responsabilidad y el compromiso social de los ciudadanos facilitando la comprensión de hechos y la toma de decisiones. En este sentido, se solicita a los informantes que seleccionen tres aspectos de la lista siguiente en los que las matemáticas pueden ayudar a opinar y a actuar con conocimiento de causa: informaciones de los medios de comunicación; datos estadísticos, como por ejemplo sondeos electorales; comprensión del espacio, por ejemplo relacionado con las redes de medios de transporte; demografía; movimientos migratorios; microeconomía y macroeconomía; cambio climático; gestión de residuos en el mundo; alfabetización; culturas diferentes de la propia; u otros aspectos.

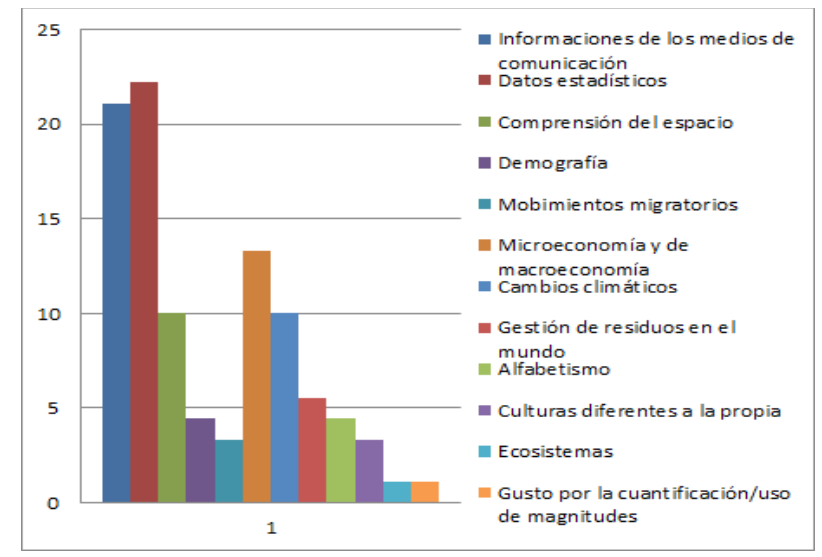

Figura 5. Responsabilidad y compromiso social

A partir de los datos de la figura 5 se deduce que para formar ciudadanos más responsables y comprometidos socialmente, el aspecto que más debería tener en cuenta el profesorado es trabajar la educación matemática a partir de una y para una mejor comprensión de las informaciones de los medios de comunicación, incluyendo los datos estadísticos que se publican en dichos medios.

\section{Globalización e interdisciplinariedad}

En este ítem se indaga en la opinión de los informantes acerca de la frecuencia en la que la educación matemática debería ser globalizada e interdisciplinar en la escuela, considerando que el mundo es globalizado e interdisciplinar.

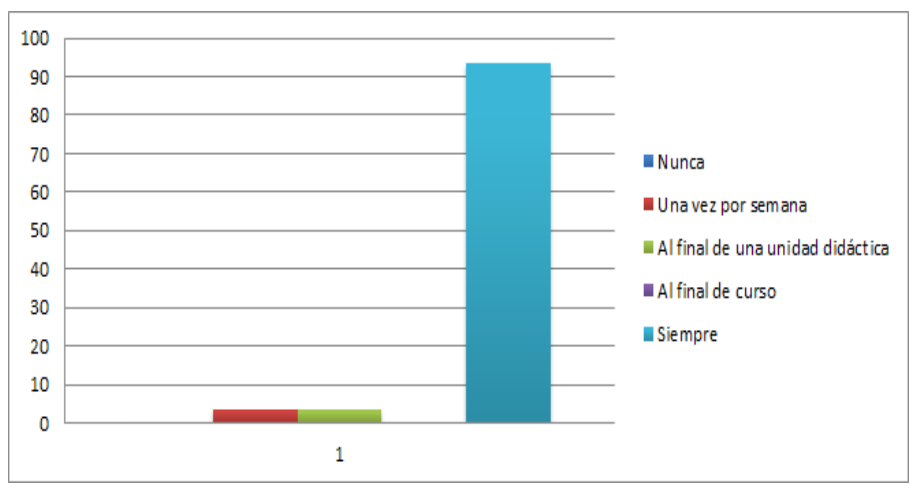

Figura 6. Globalización e interdisciplinariedad 
Como se muestra en la figura 6, los informantes han coincidido de forma muy mayoritaria en que el profesorado debería estar capacitado para trabajar en todo momento la educación matemática de manera globalizada e interdisciplinar.

\section{Cultura general}

Otro dato importante para avanzar hacia la caracterización de un modelo de formación inicial del profesorado de matemáticas en conexión con la sostenibilidad es el papel que ejerce el nivel cultural general en la competencia profesional para enseñar matemáticas.

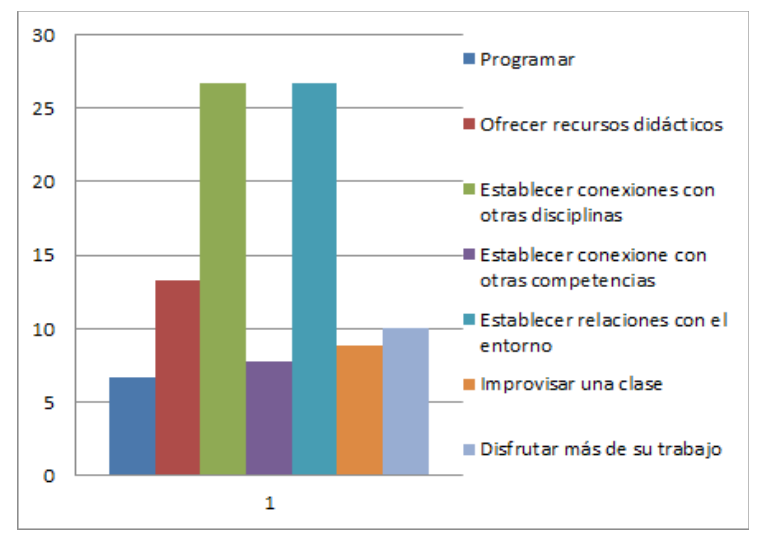

Figura 7. Cultura general

Las respuestas de los participantes del estudio han indicado de forma mayoritaria que el profesorado tendría que estar capacitado para aprovechar su cultura general como recurso de aula para establecer conexiones con otras disciplinas y con el entorno.

\section{Formación no académica}

Se ha pretendido también recoger la opinión de los informantes acerca de los tipos de actividades de formación no académica que permiten al profesorado mantener actualizada su capacidad para trabajar la educación matemática en conexión con la sostenibilidad. Para ello, se les ha solicitado que seleccionaran tres tipos de actividades de la lista siguiente: mantenerse al día de las noticias de actualidad; participar en tertulias con compañeros y amigos sobre temas de actualidad; relacionarse con gente de edades y grupos sociales y profesionales diferentes; viajar; leer novela histórica; leer artículos de opinión; u otras.

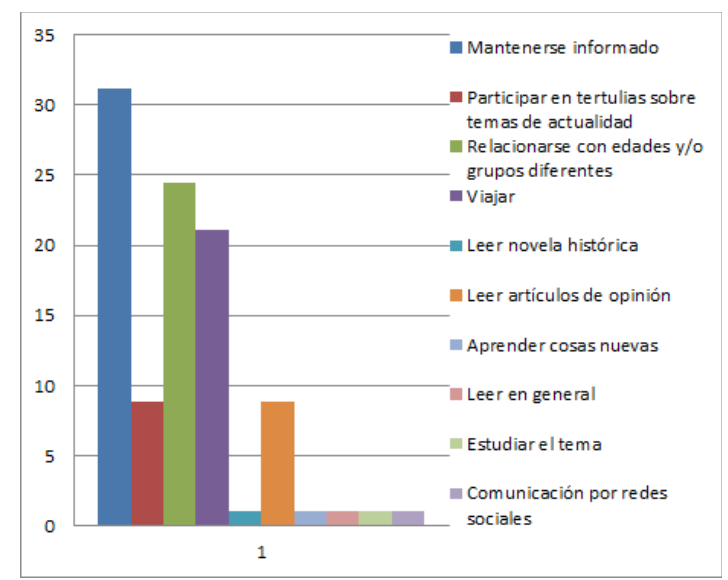

Figura 8. Formación no académica 
De acuerdo con las respuestas obtenidas, el profesorado debería estar capacitado sobre todo para mejorar día a día la formación no académica conociendo otras opiniones, contextos y cultura, además de aprovechar su formación no académica como recurso de aula para establecer conexiones con otras disciplinas y con el entorno.

\section{Criterios de concreción del curriculum de matemáticas}

Se han proporcionado a los informantes diversos criterios que el profesorado debería tener presente para concretar el curriculum en el aula: el proyecto educativo de centro (PEC), que entre otras cosas señala qué y de qué manera enseñar a los estudiantes; el pensamiento del maestro sobre qué y de qué manera enseñar a los estudiantes; los recursos disponibles en el centro; la conexión de la enseñanza escolar con el medio; las características del grupo clase; o bien las características de cada estudiante. En la figura 9 se muestran los resultados obtenidos a partir de la ordenación de dichos criterios, de menos importante (1) a más importante (6):

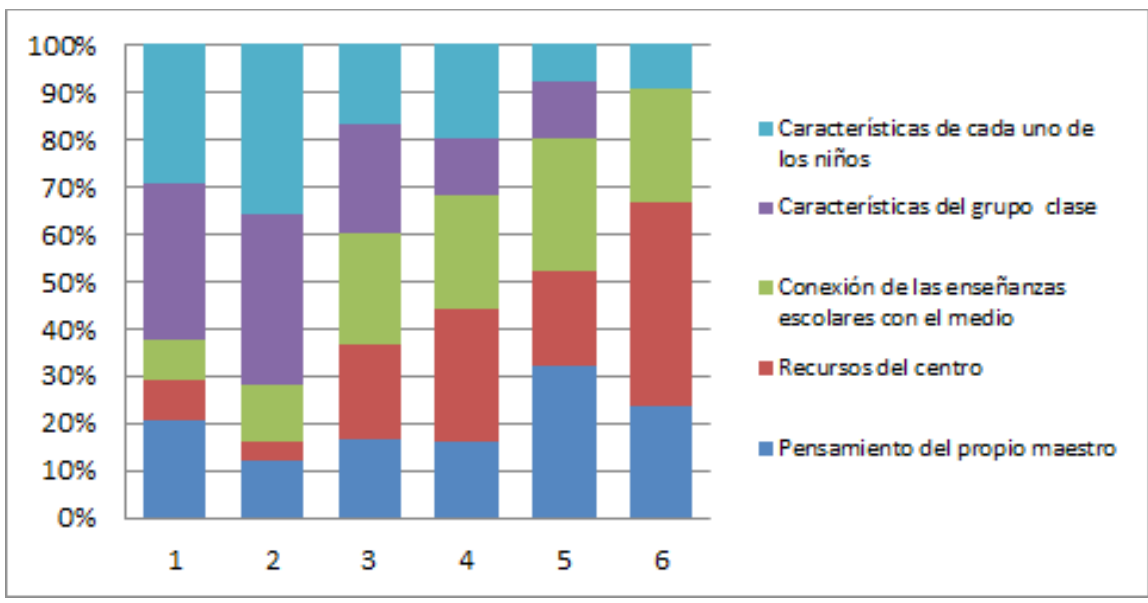

Figura 9. Criterios de concreción del curriculum

Según las opiniones de los informantes que se muestran en la figura 9, para concretar el curriculum de matemáticas, el profesorado debería saber apreciar y aprovechar las características de cada estudiante y del grupo clase para poder establecer después conexiones entre la enseñanza escolar y el medio.

\section{Competencias básicas del curriculum}

En el curriculum de Educación Primaria vigente se establecen 8 competencias: comunicativa, lingüística y audiovisual; artística y cultural; tratamiento de la información y competencia digital; matemática; aprender a aprender; autonomía e iniciativa personal; conocimiento e interacción con el mundo físico; social y ciudadana. En la figura 10 se muestran las respuestas de los informantes acerca de las competencias en las que el profesorado debería estar especialmente preparado para trabajar la educación matemática en conexión con la sostenibilidad, a partir de su ordenación desde la más importante (1) a la menos importante (8).

De los resultados obtenidos se desprende que los informantes han considerado que el profesorado debería estar capacitado principalmente para las matemáticas y para comunicarlas a los estudiantes, además de para conocer e interaccionar con el medio y aportar los aprendizajes que se desprendan al aula de matemáticas. 


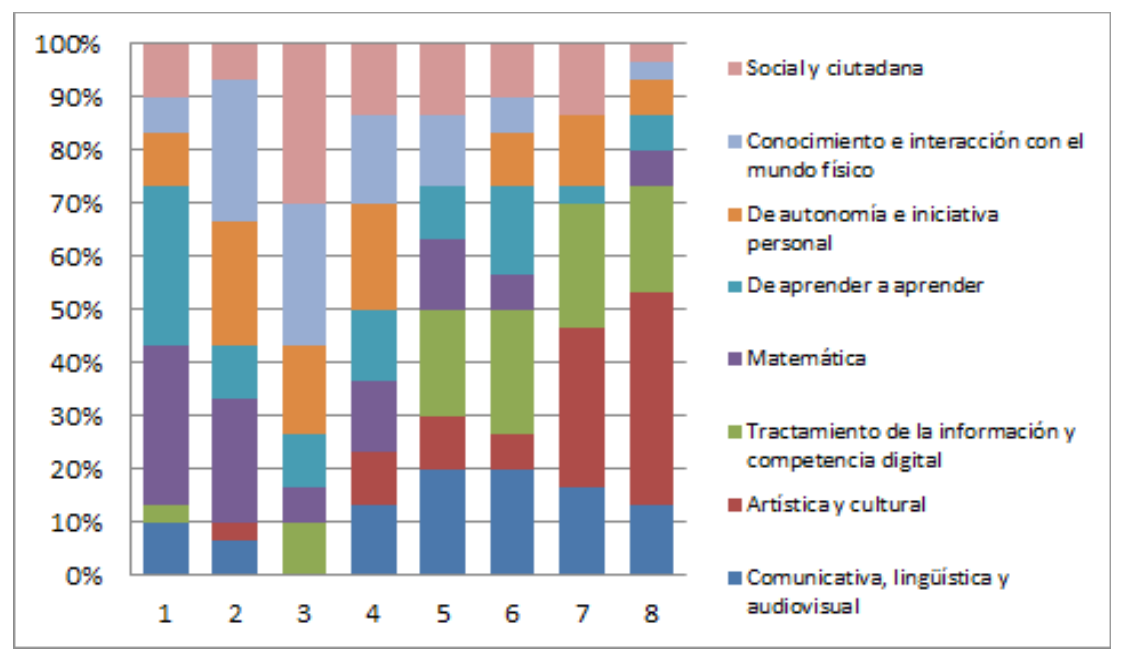

Figura 10. Competencias básicas

\section{Ámbitos del curriculum}

En el curriculum de Educación Primaria establecido por la Generalidad de Cataluña se distinguen 5 ámbitos formativos: lenguas; conocimiento del medio natural, social y cultural; educación artística; educación para el desarrollo personal y la ciudadanía; y educación física. Se ha solicitado a los participantes en el estudio que ordenaran de más importante (1) a menos importante (5) los ámbitos en los que el profesorado debería estar especialmente preparado para trabajar la educación matemática en conexión con la sostenibilidad.

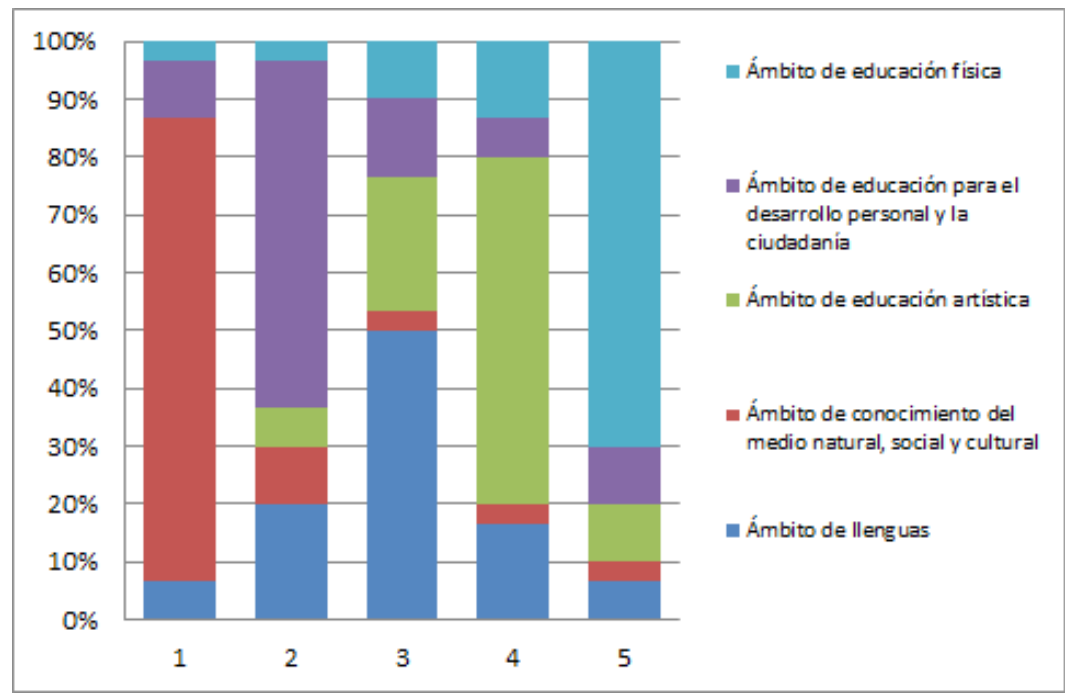

Figura 11. Ámbitos del currículum

Según las respuestas obtenidas, el profesorado debería formarse en todos los ámbitos de conocimiento, pero muy especialmente en el del conocimiento del medio natural, social y cultural.

\section{Estándares de contenidos matemáticos}

El Consejo Nacional de Profesores de Matemáticas de Estados Unidos (NCTM, 2000) ha establecido 5 estándares de contenidos matemáticos que todos los estudiantes tendrían que tener la oportunidad de aprender: números y operaciones; álgebra; 
geometría; medida; y análisis de datos y probabilidad. De nuevo, se ha solicitado a los informantes que ordenaran de más importante (1) a menos importante (5) los contenidos en los que el profesorado debería estar especialmente preparado para trabajar la educación matemática en conexión con la sostenibilidad.

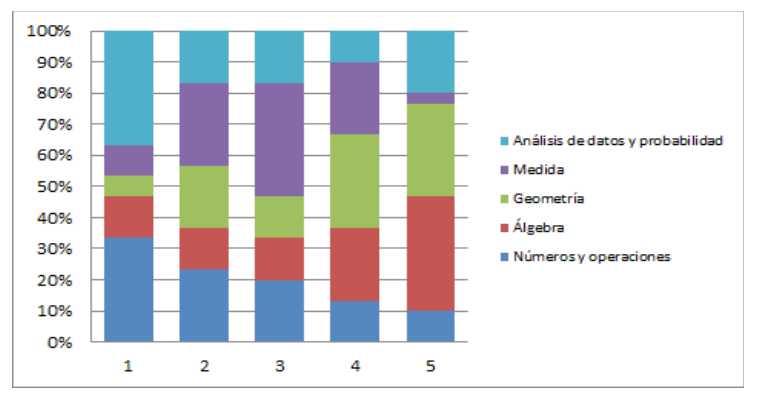

Figura 12. Estándares de contenido matemático

Los datos de la figura 12 muestran que el profesorado debería trabajar todos los contenidos matemáticos señalados en el currículum de Educación Primaria, especialmente los relacionados con numeración y cálculo y análisis de datos y probabilidad.

\section{Estándares de procesos matemáticos}

El NCTM (2000) establece también cinco procesos matemáticos para trabajar los distintos contenidos: resolución de problemas; razonamiento y prueba; comunicación; conexiones y representación. En la figura 13 se muestra la opinión de los informantes acerca del nivel de importancia de cada proceso para trabajar la educación matemática en conexión con la sostenibilidad, desde el más importante (1) hasta el menos importante (5).

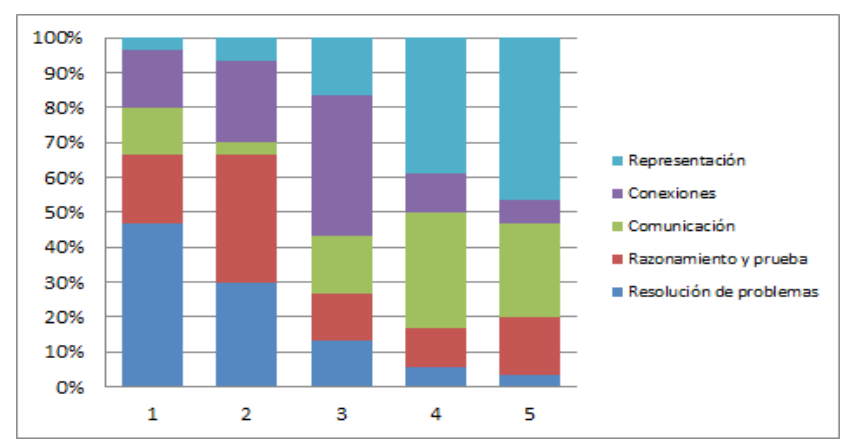

Figura 13. Estándares de proceso matemático

Como se muestra en la figura 13, los informantes han considerado que es necesario trabajar todos los procesos matemáticos señalados en el currículum de Educación Primaria, especialmente los relacionados con resolución de problemas y razonamiento y prueba.

\section{Creatividad y procesos matemáticos}

Otro aspecto en el que se ha indagado es el papel que juega la creatividad en los distintos procesos matemáticos. Para ello, se ha solicitado a los informantes que seleccionaran los dos procesos en los que, a su modo de ver, la creatividad tiene un rol más importante. 


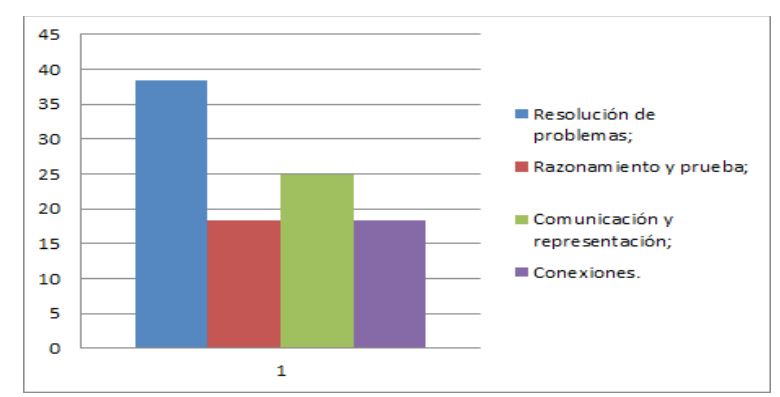

Figura 14. Creatividad y procesos matemáticos

Según las opiniones de los informantes, el profesorado debería estar capacitado para impulsar la creatividad de los estudiantes para mejorar sus resultados en todos los procesos matemáticos, sobre todo en la resolución de problemas y en el razonamiento y la prueba.

\section{Conocimiento de las normativas y documentos científicos y didácticos}

Este ítem del Cuestionario EMS pretende analizar las opiniones de los informantes acerca de los conocimientos que debería tener el profesorado sobre determinados documentos normativos y de contenido científico o didáctico para poder desarrollar con más garantías de éxito su trabajo. En este sentido, se ha solicitado a los participantes que ordenaran diversos tipos de normativas y documentos desde el que consideran más importante (1) al menos importante (4): normativas gubernamentales; normativas del mismo centro escolar; publicaciones sobre investigación en educación matemática y educación para la sostenibilidad; o bien publicaciones sobre experiencias docentes en educación matemática.

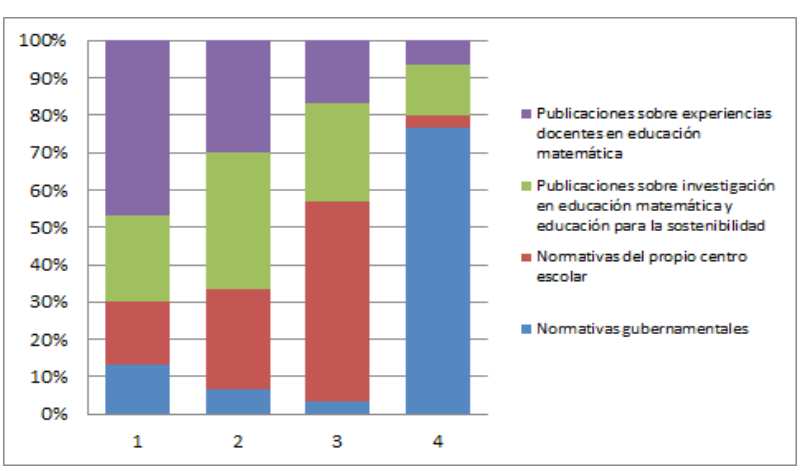

Figura 15. Normativas y documentos científico-didácticos

Los informantes consideran que, principalmente, el profesorado debería extraer información de publicaciones sobre investigación en educación matemática y educación para la sostenibilidad aplicable a su realidad profesional, además de extraer también conocimientos de publicaciones sobre experiencias docentes en educación matemática aplicable a su realidad profesional.

\section{Aprendizaje competencial}

El último ítem del cuestionario se centra en las bases del aprendizaje competencial. Algunas de estas bases se centran en el saber (conocimiento), el saber hacer (capacidades), el saber ser (habilidades), el saber estar (actitudes) y el saber actuar (movilizar recursos personales), y se pretende conocer las opiniones de los informantes sobre otros aspectos que añadirían para fortalecer la conexión entre la 
educación matemática y la sostenibilidad o bien si prescindirían de alguno de los mencionados. En la figura 16 se muestran los datos obtenidos:

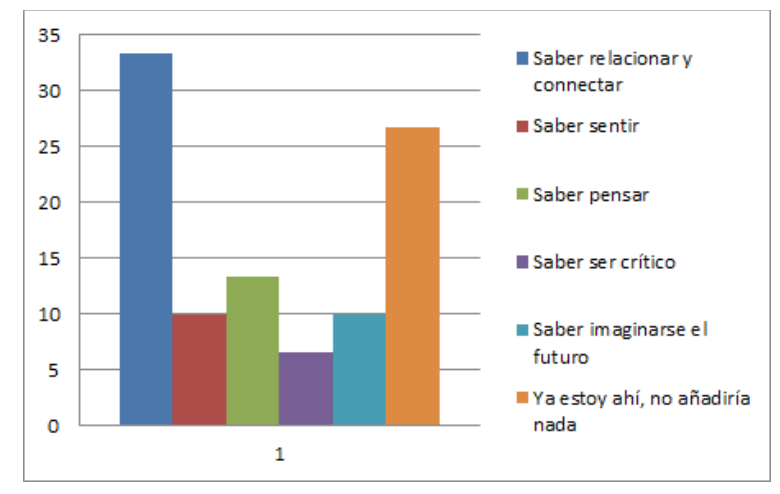

Figura 16. Bases del aprendizaje competencial

En el gráfico de la figura 16 se muestra que los informantes han considerado que el profesorado debería estar capacitado para fomentar el saber (conocimiento), el saber hacer (capacidades), el saber ser (habilidades), el saber estar (actitudes) y el saber actuar (movilizar recursos personales), además de fomentar el saber relacionar y el saber conectar, el saber sentir, el saber pensar, el saber ser crítico y el saber imaginarse el futuro.

A partir de estos datos, Calabuig (2017) ha definido veinte elementos de un perfil de maestro de matemáticas en conexión con la sostenibilidad:

1. Establecer él mismo y hacer establecer al niño una buena relación con las matemáticas a partir de la esencia de la dicha disciplina.

2. Establecer él mismo y hacer establecer al niño una buena relación entre las matemáticas y el mundo.

3. Mejorar día a día el nivel de cultura general y de formación no académica propia y de sus alumnos que pueda surgir de abrir la mirada al mundo y a su diversidad.

4. Aprovechar la cultura general y la formación no académica como recurso de EDM en el momento de establecer conexiones con otras disciplinas y el entorno.

5. Trabajar en todo momento la EDM de manera globalizada e interdisciplinaria.

6. Trabajar la formación integral del niño.

7. Dar una buena base de EDM a los niños que les vaya a ser útil en otros momentos formativos.

8. Trabajar EDM para mejorar la comprensión de las informaciones publicadas en los medios de comunicación.

9. Impulsar la creatividad de los niños para mejorar sus resultados en todos los procesos matemáticos, sobretodo en la resolución de problemas.

10. Respetar, hacer respetar, valorar, combinar e impulsar personalmente y académicamente diferentes maneras de razonar un mismo hecho matemático.

11. Reconocer la importancia del lenguaje para la construcción del pensamiento, tanto socialmente como individualmente, y trabajar en este sentido. 
12. Comprender y apreciar las matemáticas en su esencia disciplinar y en su papel como agente de cambio, tanto social como cultural, para transmitirlo a los estudiantes.

13. Comprender y apreciar la sostenibilidad por lo que conlleva de responsabilidad y compromiso social, para transmitirlo a los estudiantes.

14. Trabajar en todos los ámbitos del conocimiento, pero especialmente en el de las matemáticas en conexión con el medio natural, social y cultural.

15. Comprender y apreciar todos los contenidos matemáticos del currículum de educación primaria.

16. Trabajar todos los procesos matemáticos del currículum de educación primaria.

17. Saber ser crítico con el currículum si la situación educativa lo requiere.

18. Seguir con la propia formación permanente de manera autónoma.

19. Extraer información de publicaciones sobre investigación y experiencias docentes en EDM y EDS aplicable a su propia realidad profesional.

20. Saber (conocimiento), saber hacer (capacidades), saber ser (habilidades), saber estar (actitudes), saber actuar (movilizar recursos personales), saber sentir (emociones) y saber imaginarse el futuro (prever).

De acuerdo con estos datos y con los antecedentes expuestos, en el siguiente apartado se intenta avanzar hacia la caracterización de un modelo de formación inicial del profesorado de matemáticas que contribuya a dar respuesta a los retos sociales contemporáneos, en sintonía con los avances de la investigación en sostenibilidad.

\section{Consideraciones finales: hacia la caracterización de un modelo de formación inicial del profesorado de matemáticas en conexión con la sostenibilidad}

A partir de los datos obtenidos y de los referentes teóricos expuestos, se han definido (en una primera instancia) las siguientes cinco dimensiones que configuran nuestro modelo:

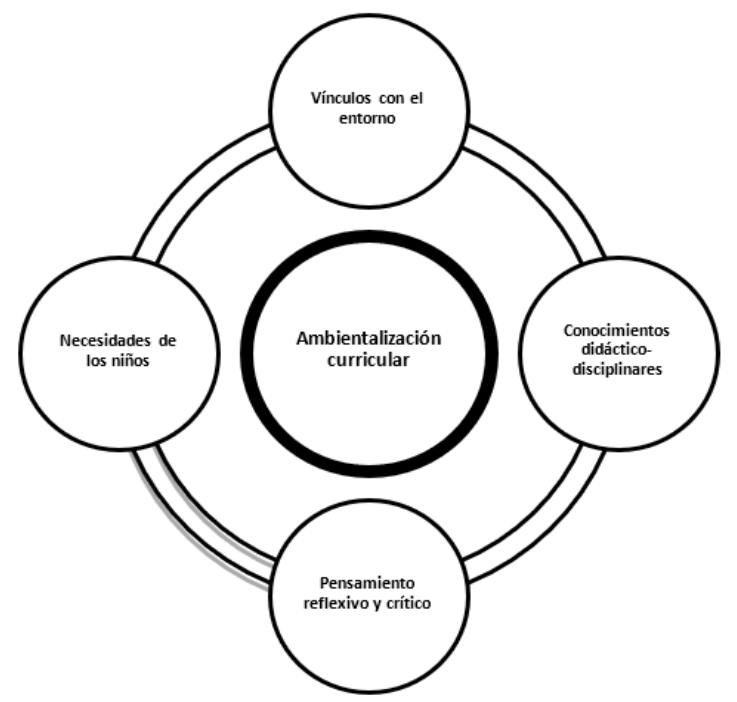

Figura 17. Dimensiones para la caracterización de un modelo de formación inicial del profesorado de matemáticas de Educación Primaria en conexión con la sostenibilidad. 
A continuación se describen brevemente estas cinco dimensiones:

1. Vínculos con el entorno: esta dimensión se refiere a que, durante la formación inicial, los futuros maestros deberían aprender a mirar profesionalmente el medio natural, social y cultural para determinar lo que es relevante y trasladarlo a las situaciones de enseñanza, como por ejemplo lo que puede aportar la cultura general, las informaciones que aparecen en los medios de comunicación o bien el desarrollo sostenible y su incidencia en la responsabilidad y compromiso social. Con ello, se debería conseguir que los aprendices para maestro comprendieran el papel que puede jugar la educación matemática como agente de cambio, tanto social como cultural.

2. Conocimientos didáctico-disciplinares: esta dimensión se refiere a que, durante la formación inicial, los futuros maestros deberían establecer una buena relación con las matemáticas a partir de la naturaleza de la disciplina, comprendiendo en profundidad qué conocimientos enseñar (contenidos y procesos matemáticos) y cómo enseñarlos. Deberían también comprender la importancia de la formación permanente para su desarrollo profesional continuo, a partir de distintas fuentes como publicaciones científicas sobre educación matemática y educación para la sostenibilidad, etc.

3. Pensamiento reflexivo y crítico: esta dimensión se refiere a que, durante la formación inicial, los futuros maestros deberían desarrollar habilidades para aprender a enfrentarse a la propia actuación, a la propia realidad, a los propios problemas y a las propias circunstancias y a llevar a cabo una reflexión continuada de su quehacer diario. El objetivo es que los futuros maestros lleguen por sí mismo no solamente a descubrir los aspectos que quiere o debe cambiar o mejorar sino a buscar soluciones y a evaluarlas por sí mismo. Entran así en una dimensión autorreguladora, de gran importancia para el aprendizaje autónomo.

4. Necesidades de los niños: esta dimensión se refiere a que, durante la formación inicial, los futuros maestros deberían ir construyendo un perfil docente propio que les permita centrarse en las necesidades reales de los niños, más allá de las modas y tendencias pasajeras que en ocasiones se venden como innovaciones didácticas. En este sentido, por ejemplo, los aprendices para maestro deberían adquirir estrategias para que, desde una formación integral, los niños establezcan una buena relación con las matemáticas a partir de la naturaleza de la disciplina, que conozcan las conexiones de las matemáticas con el entorno, o bien que puedan desarrollar su creatividad.

5. Ambientalización curricular: esta dimensión se ha colocado en el centro del modelo porque vehicula el resto y se refiere a que, durante la formación inicial, los futuros maestros de matemáticas deberían formarse a partir de un plan de estudios elaborado con base a criterios de desarrollo sostenible que les permita, por ejemplo, aprender a seleccionar los conocimientos del currículum con criterios de sostenibilidad o bien afrontar con naturalidad y normalidad la complejidad del mundo, en constante evolución.

A partir de los datos aportados, será necesario diseñar nuevos estudios que permitan analizar con precisión el grado de ambientalización curricular del Grado de Maestro en general y de la formación inicial de maestros de matemáticas en particular (por 
ejemplo, a partir de la definición de indicadores específicos para cada dimensión y del diseño, construcción y validación de instrumentos de análisis), junto con diseñar planes de formación específicos para ambientalizar, si es necesario, dichos estudios.

\section{Referencias}

Alsina, Á. (2007). El aprendizaje reflexivo en la formación permanente del profesorado: un análisis desde la didáctica de la matemática. Educación Matemática, 19(1), 99-126.

Alsina, Á. (2010). El aprendizaje reflexivo en la formación inicial del profesorado: un modelo para aprender a enseñar matemáticas. Educación Matemática, 22(1), 149-166.

Barañano, M. y Comisión RSU (2011). La responsabilidad social de la universidad y el desarrollo sostenible. Madrid: Secretaría General Técnica, Ministerio de Educación.

Calabuig, T. (2017). Connexions entre educació matemàtica i educació per a la sostenibiltat: definició d'un perfil de mestre de matemàtiques. Tesis doctoral. Universitat de Girona. Girona. Recuperado de http://hdl.handle.net/10803/401745

Calabuig, T., Alsina, Á., y Geli, A. M. (2017). Definición de un perfil de maestro de matemáticas en concordancia con la educación para la sostenibilidad. Enseñanza de las Ciencias, № Extra 2017, 281-286.

Copernicus-Alliance. (2016). Copernicus-Alliance. Recuperado de http://www.copernicus-alliance.org/

CRUE_CADEP. (2016). Directrices para la introducción de la sostenibilidad en el currículum. Recuperado de https://www.crue.org/Documentos \%20compartidos/Declaraciones/Directrices_Sosteniblidad_Crue2012.pdf

Esteve, O., Melief, K., y Alsina, Á. (2010). Creando mi profesión: una propuesta para el desarrollo profesional de profesorado. Barcelona: Octaedro.

Freudenthal, H. (1991). Revisiting Mathematics Education. Boston, Dordrecht: Kluwer Academic Publishers.

Geli, A. M., Junyent, M., Medir, R., y Padilla, F. (2006). L'ambientalització curricular en l'ensenyament obligatori: una proposta de definició, caracterització i estratègies. Barcelona: Departament de Medi Ambient i Habitatge, Generalitat de Catalunya.

González, E. J. (2012). La ambientalización del currículum escolar: breve recuento de una azarosa historia. Profesorado. Revista de Currículum y Formación del Profesorado, 16(2), 15-24.

Goñi, J. M. (2010). ¿Cómo hacer frente a la complejidad de las competencias desde el diseño curricular? Un problema de ingeniería curricular. Aula de Innovación Educativa, 17, 6-11.

Gutiérrez-Pérez, J. y Perales-Palacios, F. J. (2012). Ambientación curricular y sostenibilidad. Nuevos retos de profesionalización docente. Profesorado. Revista de Currículum y Formación del Profesorado, 16(2), 5-14. 
Junyent, M., Geli, A. M., y Arbat, E. (2003). Ambientalización curricular de los estudios superiores. 2. Proceso de caracterización de la ambientalización curricular de los estudios superiores. Girona: Universitat de Girona-RED ACES.

Kilpatrick, J. (1988). Change and stability in research in mathematics education. ZDM, 5, 202-204.

Korthagen, F. A. J. (2001). Linking practice and theory: The pedagogy of realistic teacher education. London: Lawrence Erlbaum Associates.

Korthagen, F. (2010). La práctica, la teoría y la persona en la formación del profesorado. Revista Interuniversitaria de Formación del Profesorado, 68 (24,2), 83-102.

Llinares, S. (2008). Agendas de investigación en Educación Matemática en España. Una aproximación desde "ISI-web of knowledge". En R. Luengo, B. Gómez, M. Camacho y L. J. Blanco (Eds.), Investigación en Educación Matemática XII (pp. 25-54). Badajoz: Sociedad Extremeña de Educación Matemática "Ventura Reyes Prósper" y SEIEM.

Llinares, S. (2012). Construcción de conocimiento y desarrollo de una Mirada profesional para la práctica de enseñar matemáticas en entornos en línea. AIEM. Avances de Investigación en Educación Matemática, 2, 53-70.

Llinares, S. (2018). Escribir narrativas. De observar a mirar profesionalmente. En L. J. Rodríguez-Muñiz, L. Muñiz-Rodríguez, A. Aguilar-González, P. Alonso, F. J. García García, y A. Bruno (Eds.), Investigación en Educación Matemática XXII (pp. 39-50). Gijón: SEIEM.

Melief, K., Tigchelaar, A., y Korthagen, F., (2010). Aprender de la práctica. En O. Esteve, K. Melief y Á. Alsina (Eds.), Creando mi profesión. Una propuesta para el desarrollo profesional del profesorado (pp. 19-38). Barcelona: Octaedro.

NCTM (2000). Principles and Standards for School Mathematics.Reston, VA: NCTM.

Robinson, N. A., Astorga, L. E., Oksanen, T., y Trigo, E. J. (1993). Agenda 21: Earth's Action Plan. Gland (Suïssa). Recuperado de https://sustainabledevelopment.un.org/outcomedocuments/agenda21

Schmittau, J. (2003). Cultural-Historical Theory and Mathematics Education. A Vygotsky's Educational Theory in Cultural Context. Cambridge: Cambridge University Press.

UNESCO. (2004). United Nations Decade of Education for Sustainable Development 2005-2014: Draft International Implementation Scheme. UNESCO. 